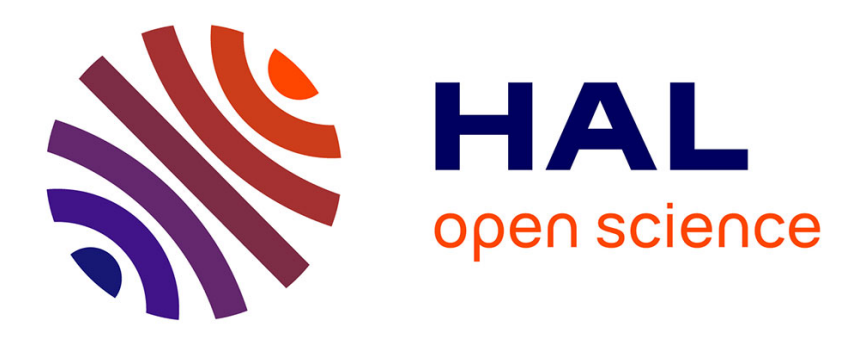

\title{
Axiomatic properties of geo-logarithmic price indexes
}

Marco Fattore

\section{To cite this version:}

Marco Fattore. Axiomatic properties of geo-logarithmic price indexes. Econometrics, 2009, 10.1016/j.jeconom.2009.11.004 . hal-00647881

\section{HAL Id: hal-00647881 \\ https://hal.science/hal-00647881}

Submitted on 3 Dec 2011

HAL is a multi-disciplinary open access archive for the deposit and dissemination of scientific research documents, whether they are published or not. The documents may come from teaching and research institutions in France or abroad, or from public or private research centers.
L'archive ouverte pluridisciplinaire HAL, est destinée au dépôt et à la diffusion de documents scientifiques de niveau recherche, publiés ou non, émanant des établissements d'enseignement et de recherche français ou étrangers, des laboratoires publics ou privés. 


\section{Accepted Manuscript}

Axiomatic properties of geo-logarithmic price indexes

Marco Fattore

PII:

S0304-4076(09)00293-0

DOI:

10.1016/j.jeconom.2009.11.004

Reference:

ECONOM 3293

To appear in: Journal of Econometrics

Received date: 1 November 2007

Revised date: 16 February 2009

Accepted date: 16 November 2009

Please cite this article as: Fattore, M., Axiomatic properties of geo-logarithmic price indexes.

Journal of Econometrics (2009), doi:10.1016/j.jeconom.2009.11.004

This is a PDF file of an unedited manuscript that has been accepted for publication. As a service to our customers we are providing this early version of the manuscript. The manuscript will undergo copyediting, typesetting, and review of the resulting proof before it is published in its final form. Please note that during the production process errors may be discovered which could affect the content, and all legal disclaimers that apply to the journal pertain. 


\title{
Axiomatic properties of geo-logarithmic price indexes
}

\author{
Marco Fattore \\ Università degli Studi di Milano-Bicocca, via Bicocca degli Arcimboldi 8, 20126 \\ Milan, Italy
}

\begin{abstract}
In the axiomatic approach to composite index numbers, a list of properties is given that both price and quantity indexes should satisfy in order to assure consistent comparisons. Usually, the price index is selected first and its cofactor is consequently adopted as the (implicit) quantity index. Unfortunately, even if the price index has good axiomatic properties, its cofactor need not, so that the implicit quantity comparison may be axiomatically inconsistent. In this paper, we give a comprehensive study of a family of price indexes sharing good axiomatic properties (proportionality, commensurability, and homogeneity) together with their cofactors. This family, called geo-logarithmic, is relevant also because of the empirical circumstance that all known price indexes sharing such properties with their cofactors belong to it or can be obtained from geo-logarithmic index numbers through simple transformations. Thus, the geo-logarithmic family seems to play a central role when the joint consistency of price and quantity comparisons is concerned.
\end{abstract}

Key words: Price index, Price comparison, Implicit quantity index, Cofactor, Axiomatic Index Number Theory.

PACS: C43

\section{Introduction}

In the axiomatic approach to composite index numbers (Balk, 1995), price and quantity indexes are dually linked since their product has to decompose the value index in a multiplicative way. In practice, the price index is often given some prominence, so that its formula is selected first and its cofactor is de facto chosen as the quantity index assuring for the value index decomposition to hold. This fact introduces a small "symmetry breaking" in the duality

Email address: marco.fattore@unimib.it (Marco Fattore). 
of price and quantity indexes, which is reasonable in itself, since often price comparison is the main interest. Unfortunately such an induced asymmetry can also have unexpected consequences Usually no requirement is given connecting the choice of the price index to the properties actually satisfied by its cofactor, so that the former is very often selected irrespective of the axiomatic properties fulfilled by the latter. This way, on a logical ground the cofactor is given almost a residual meaning and the initial small symmetry breaking results into a great difference in the roles assigned to price and quantity indexes. Most important, in general few axiomatic properties of the price index are inheritated by its cofactor (e.g. commensurability and basis reversibility), so that even if a price index satisfies many axioms, its cofactor could be very unsatisfactory from an axiomatic point of view. It is noticeable that among the axioms that a cofactor could violate even if the price index does satisfy them, there are some essential requirements, such as proportionality and homogeneity. Using a quantity index which is not proportional (or homogeneous) directly rises the question of the meaning of the quantity comparison, since the lacking of proportionality or homogeneity is a serious drawback, in an axiomatic setting. For example, how can a quantity comparison be interpreted if, say, quantity doubles over time, but the quantity index does not reveal it? Even if it is the price comparison to be primarily concerned, when a price index is chosen, implicitly a quantity comparison is also performed. But if the latter is not axiomatically consistent, also the consistency of the former (and the consistency of the value index decomposition) could be perhaps argued. Thus, as far as the joint consistency of price and quantity comparison is concerned, the axiomatic properties of the cofactor of a price index should be taken into account, when the price index is being chosen. As stated above, usually this is not the case and also from a theoretical point of view, little attention seems to have been paid to this kind of problem.

In the axiomatic framework, as it is usually presented, only the fulfillment of the factor reversibility axiom assures the cofactor to satisfy (with respect to quantities) all the properties fulfilled by the price index itself (with respect to prices), since in that case the cofactor and the price index share the same functional form. Nevertheless, the factor reversibility axiom is a very strong requirement and some argue that it is not an essential property (IMF, 2004); moreover, most of the index currently used or studied do not fulfill it. In order to assure the joint consistency of both price and quantity comparisons, a possible alternative to restricting ourselves to factor reversible price indexes, is to search for a class of price index formulas satisfying at least a subset of fundamental axiomatic properties together with their cofactors. This way, even if some priority is given to the price index, the properties of the cofactor are $a b$ initio explicitly taken into account. Motivated by the search for such a class, the geo-logarithmic family of price indexes was proposed in the early '90s, by the Italian statistician Marco Martini (Martini, 1992a,b). Its peculiarity is that all of its members do share the essential axiomatic properties of propor- 
tionality, commensurability and homogeneity together with their cofactors, so that both prices and quantities can be jointly compared in a consistent way. But perhaps even more remarkably, among price indexes studied in literature and applied in practice, the only ones sharing such three axiomatic properties with their cofactors belong to the geo-logarithmic family or can be obtained from geo-logarithmic indexes, by means of simple transformations, e.g. taking their basis or factor antitheses or their crossings (Martini, 2003). Thus, the geo-logarithmic family seems to have a peculiar role, when one is interested in the joint consistency of price and quantity comparisons. Notwithstanding this fact, a comprehensive study of its properties still lacks and the present paper aims at filling this gap; at the same time, it gives a review of already existing results and fixes some mistakes that can be found in other references. The paper is organized as follows: Section 2 gives a brief review of the axiomatic approach to price indexes; Section 3 provides the formal definition of the geo-logarithmic family; Sections 4 and 5 discuss the properties of geologarithmic price indexes; Sections 6 discusses the properties of the cofactors of geo-logarithmic price indexes; Section 7 studies the relationship between the geo-logarithmic family and the family of Diewert's superlative price indexes; Section 8 provides some final comments and points out some open issues, needing further research; Appendices A to F collect some computational details needed in the paper.

\section{Elements of Axiomatic Index Number Theory}

Let $\boldsymbol{p}_{a}, \boldsymbol{p}_{b}, \boldsymbol{q}_{a}$ and $\boldsymbol{q}_{b}$ be four $n$-dimensional vectors $(n \geq 2)$ of strictly positive components, representing the prices and the quantities of the same $n$ goods in situation $a$ and in situation $b$ ( $b$ will be always considered as the basis, i.e. the reference situation, for the comparison). We indicate with $p_{a i}, p_{b i}, q_{a i}$ and $q_{b i}(i=1, \ldots, n)$ the $i$-th component of $\boldsymbol{p}_{a}, \boldsymbol{p}_{b}, \boldsymbol{q}_{a}$ and $\boldsymbol{q}_{b}$. The ratio

$$
V=\frac{\sum_{i=1}^{n} p_{a i} q_{a i}}{\sum_{i=1}^{n} p_{b i} q_{b i}}
$$

is called the value index between $a$ and $b$. The aim of price and quantity index theory is to decompose the value index as the product of two strictly positive functions

$$
V=P\left(\boldsymbol{p}_{a}, \boldsymbol{p}_{b}, \boldsymbol{q}_{a}, \boldsymbol{q}_{b}\right) \cdot Q\left(\boldsymbol{p}_{a}, \boldsymbol{p}_{b}, \boldsymbol{q}_{a}, \boldsymbol{q}_{b}\right)
$$

where $P$ accounts for the variation of the prices and $Q$ accounts for the variation of the quantities between situations $a$ and $b$. In the axiomatic setting, the basic idea is to identify a set of axioms that a function of prices and quantities has to satisfy, in order to be accepted as a price or a quantity index and then to derive or look for formulas satisfying them. 


\subsection{The axioms}

The literature about Axiomatic Index Number Theory is very wide (Eichhorn \& Voeller, 1976a,b, 1990; Balk, 1995; Krstcha, 1988) but there is no universal agreement on the axiomatic properties needed for a formula to be considered as an index (IMF, 2004). For this reason, in this paper we focus on a set of six axioms that are generally accepted, even if some authors do criticize some of them (particularly, axioms (IV) and (VI) (Diewert, 1992; Reinsdorf \& Dorfman, 1999; IMF, 2004)). The list is given below:

(I) Proportionality axiom. Let $\alpha$ be a strictly positive real number, then

$$
P\left(\alpha \boldsymbol{p}_{b}, \boldsymbol{p}_{b}, \boldsymbol{q}_{a}, \boldsymbol{q}_{b}\right)=\alpha .
$$

(II) Commensurability axiom. Let $U$ be a $n \times n$ diagonal matrix of strictly positive weights, then

$$
P\left(U \boldsymbol{p}_{a}, U \boldsymbol{p}_{b}, U^{-1} \boldsymbol{q}_{a}, U^{-1} \boldsymbol{q}_{b}\right)=P\left(\boldsymbol{p}_{a}, \boldsymbol{p}_{b}, \boldsymbol{q}_{a}, \boldsymbol{q}_{b}\right) .
$$

(III) Homogeneity axiom. Let $\alpha$ and $\beta$ be two strictly positive real numbers, then

$$
P\left(\alpha \boldsymbol{p}_{a}, \beta \boldsymbol{p}_{b}, \boldsymbol{q}_{a}, \boldsymbol{q}_{b}\right)=\frac{\alpha}{\beta} P\left(\boldsymbol{p}_{a}, \boldsymbol{p}_{b}, \boldsymbol{q}_{a}, \boldsymbol{q}_{b}\right) .
$$

(IV) Monotonicity axiom. Let $\hat{\boldsymbol{p}}_{a}>\boldsymbol{p}_{a}$, then

$$
P\left(\hat{\boldsymbol{p}}_{a}, \boldsymbol{p}_{b}, \boldsymbol{q}_{a}, \boldsymbol{q}_{b}\right) \geq P\left(\boldsymbol{p}_{a}, \boldsymbol{p}_{b}, \boldsymbol{q}_{a}, \boldsymbol{q}_{b}\right) ;
$$

similarly, let $\hat{\boldsymbol{p}}_{b}>\boldsymbol{p}_{b}$, then

$$
P\left(\boldsymbol{p}_{a}, \hat{\boldsymbol{p}}_{b}, \boldsymbol{q}_{a}, \boldsymbol{q}_{b}\right) \leq P\left(\boldsymbol{p}_{a}, \boldsymbol{p}_{b}, \boldsymbol{q}_{a}, \boldsymbol{q}_{b}\right)
$$

where $\hat{\boldsymbol{p}}_{s}>\boldsymbol{p}_{s}$ if $\hat{p}_{s i} \geq p_{s i}$ for all $i=1, \ldots, n$ and $j$ exists such that $\hat{p}_{s j}>p_{s j}$, with $s=a, b$.

(V) Basis reversibility axiom. Let the situations $a$ and $b$ be exchanged and let $a$ be taken as the basis for the comparison, then

$$
P\left(\boldsymbol{p}_{b}, \boldsymbol{p}_{a}, \boldsymbol{q}_{b}, \boldsymbol{q}_{a}\right)=\frac{1}{P\left(\boldsymbol{p}_{a}, \boldsymbol{p}_{b}, \boldsymbol{q}_{a}, \boldsymbol{q}_{b}\right)} .
$$

(VI) Factor reversibility axiom. Let the vectors $\boldsymbol{p}_{a}$ and $\boldsymbol{p}_{b}$ be exchanged with the vectors $\boldsymbol{q}_{a}$ and $\boldsymbol{q}_{b}$ respectively, then

$$
P\left(\boldsymbol{q}_{a}, \boldsymbol{q}_{b}, \boldsymbol{p}_{a}, \boldsymbol{p}_{b}\right)=\frac{V}{P\left(\boldsymbol{p}_{a}, \boldsymbol{p}_{b}, \boldsymbol{q}_{a}, \boldsymbol{q}_{b}\right)}
$$

(recall that the function $P\left(\boldsymbol{p}_{a}, \boldsymbol{p}_{b}, \boldsymbol{q}_{a}, \boldsymbol{q}_{b}\right)$ is asked to be strictly positive). 
An analogous axiomatic system holds for quantity indexes. It is obtained simply exchanging the role of price and quantity vectors in the above list of axioms (I)-(VI). In general, price index formulas used for concrete price comparisons do not fulfill all the axioms listed above (Fisher, 1922; Swamy, 1965; Balk, 1995). As a matter of fact, not all the axioms are given the same relevance: axioms (I)-(III) are retained as fundamental, while axioms (IV)-(VI) are treated as less relevant. Hence proportionality, commensurability and homogeneity can be regarded as a minimal subset of axiomatic properties that a candidate price index should satisfy to be adopted in price comparisons. Similar considerations hold for quantity indexes.

\subsection{Cofactor and correspondent of a price index}

Chosen a price index formula $P\left(\boldsymbol{p}_{a}, \boldsymbol{p}_{b}, \boldsymbol{q}_{a}, \boldsymbol{q}_{b}\right)$, two candidate quantity indexes can be naturally associated to it. The first is called the cofactor of $P$ and is defined by

$$
\operatorname{Cof} P\left(\boldsymbol{p}_{a}, \boldsymbol{p}_{b}, \boldsymbol{q}_{a}, \boldsymbol{q}_{b}\right)=\frac{V}{P\left(\boldsymbol{p}_{a}, \boldsymbol{p}_{b}, \boldsymbol{q}_{a}, \boldsymbol{q}_{b}\right)} ;
$$

the second is called the correspondent of $P$ and is defined exchanging the role of price and quantity vectors in the formula for $P$ :

$$
\operatorname{Cor} P\left(\boldsymbol{p}_{a}, \boldsymbol{p}_{b}, \boldsymbol{q}_{a}, \boldsymbol{q}_{b}\right)=P\left(\boldsymbol{q}_{a}, \boldsymbol{q}_{b}, \boldsymbol{p}_{a}, \boldsymbol{p}_{b}\right) .
$$

In general, cofactor and correspondent of the same price index differ; they coincide if and only if the price index satisfies the factor reversibility axiom. By virtue of (2), when a price index is chosen, its cofactor is de facto selected as the associated quantity index (for this reason, the cofactor is also called the implicit quantity index). As a consequence, a function $P\left(\boldsymbol{p}_{a}, \boldsymbol{p}_{b}, \boldsymbol{q}_{a}, \boldsymbol{q}_{b}\right)$ should not be accepted as a price index, irrespective of the axiomatic properties satisfied by its cofactor. In fact, as briefly discussed in the Introduction, counterexamples can be given showing that the fundamental properties of proportionality and homogeneity of the cofactor are independent from the analogous properties of the price index (Martini, 1992a).

\subsection{The antithesis operators}

We now introduce some algebraic tools that prove very useful in the subsequent analysis of the axiomatic properties of geo-logarithmic price indexes. 


\subsubsection{The Antithesis group}

The basis reversibility and the factor reversibility axioms define some internal symmetries that a price index should satisfy. Let us introduce two operators $\boldsymbol{B}$ and $\boldsymbol{F}$ whose actions are defined as:

$$
\begin{aligned}
& \boldsymbol{B P}\left(\boldsymbol{p}_{a}, \boldsymbol{p}_{b}, \boldsymbol{q}_{a}, \boldsymbol{q}_{b}\right)=\frac{1}{P\left(\boldsymbol{p}_{b}, \boldsymbol{p}_{a}, \boldsymbol{q}_{b}, \boldsymbol{q}_{a}\right)} ; \\
& \boldsymbol{F} P\left(\boldsymbol{p}_{a}, \boldsymbol{p}_{b}, \boldsymbol{q}_{a}, \boldsymbol{q}_{b}\right)=\frac{V}{P\left(\boldsymbol{q}_{a}, \boldsymbol{q}_{b}, \boldsymbol{p}_{a}, \boldsymbol{p}_{b}\right)} .
\end{aligned}
$$

$\boldsymbol{B} P$ and $\boldsymbol{F} P$ are called the basis antithesis and the factor antithesis of $P$ and the operators $\boldsymbol{B}$ and $\boldsymbol{F}$ can be called the basis antithesis operator and the factor antithesis operator respectively (Fattore \& Quatto, 2004; Fattore, 2006b). The reversibility axioms can thus be stated as invariance properties of a price index $P$ under the action of the antithesis operators:

$$
\begin{aligned}
& \boldsymbol{B} P\left(\boldsymbol{p}_{a}, \boldsymbol{p}_{b}, \boldsymbol{q}_{a}, \boldsymbol{q}_{b}\right)=P\left(\boldsymbol{p}_{a}, \boldsymbol{p}_{b}, \boldsymbol{q}_{a}, \boldsymbol{q}_{b}\right) \quad \text { (basis reversibility); } \\
& \boldsymbol{F} P\left(\boldsymbol{p}_{a}, \boldsymbol{p}_{b}, \boldsymbol{q}_{a}, \boldsymbol{q}_{b}\right)=P\left(\boldsymbol{p}_{a}, \boldsymbol{p}_{b}, \boldsymbol{q}_{a}, \boldsymbol{q}_{b}\right) \quad \text { (factor reversibility). }
\end{aligned}
$$

A direct computation shows that $\boldsymbol{B}$ and $\boldsymbol{F}$ commute (Vogt \& Barta, 1997; Fattore \& Quatto, 2004; Fattore, 2006b), thus the operator $\boldsymbol{D}=\boldsymbol{B} \circ \boldsymbol{F}=\boldsymbol{F} \circ \boldsymbol{B}$ can be defined. The identity operator $\boldsymbol{I}$ and the three operators $\boldsymbol{B}, \boldsymbol{F}$ and $\boldsymbol{D}$ form a commutative group (Miller, 1972; Tung, 1985; Bosch, 2003), whose Cayley table is given by

$$
\begin{array}{c|cccc} 
& \boldsymbol{I} & \boldsymbol{B} & \boldsymbol{F} & \boldsymbol{D} \\
\hline \boldsymbol{I} & \boldsymbol{I} & \boldsymbol{B} & \boldsymbol{F} & \boldsymbol{D} \\
\boldsymbol{B} & \boldsymbol{B} & \boldsymbol{I} & \boldsymbol{D} & \boldsymbol{F} \\
\boldsymbol{F} & \boldsymbol{F} & \boldsymbol{D} & \boldsymbol{I} & \boldsymbol{B} \\
\boldsymbol{D} & \boldsymbol{D} & \boldsymbol{F} & \boldsymbol{B} & \boldsymbol{I}
\end{array}
$$

We will refer to this group as the Antithesis group. Requiring a price index to satisfy the reversibility axioms (V) and (VI) is equivalent to requiring it to be invariant under the action of the Antithesis group or, that is the same, to be a fixed point of the operators belonging to the Antithesis group.

It is straightforward to show that the basis antithesis of a price index fulfills any of the axiomatic properties (I)-(VI) if and only if the price index itself does (Martini, 1992a). On the contrary, the only axiomatic properties that the factor antithesis inheritates from the index are those of commensurability, 
basis reversibility and, trivially, factor reversibility. Counterexamples can be given of homogeneous or monotonic price indexes with factor antitheses that do not satisfy such properties ((Fattore, 2006a) or Proposition 6 below).

\subsubsection{Structure of the factor antithesis operator}

In terms of cofactor and correspondent of a price index, the factor reversibility axiom can be trivially stated as

$$
\operatorname{Cor} P=\operatorname{Cof} P
$$

Let $\boldsymbol{H}$ and $\boldsymbol{K}$ be two operators, defined as:

$$
\begin{aligned}
& \boldsymbol{H} P=\operatorname{Cor} P \\
& \boldsymbol{K} P=\operatorname{Cof} P .
\end{aligned}
$$

The operators $\boldsymbol{F}, \boldsymbol{H}$ and $\boldsymbol{K}$, together with the identity operator $\boldsymbol{I}$, constitute a group, with the following Cayley table

$$
\begin{array}{c|cccc} 
& \boldsymbol{I} & \boldsymbol{H} & \boldsymbol{K} & \boldsymbol{F} \\
\hline \boldsymbol{I} & \boldsymbol{I} & \boldsymbol{H} & \boldsymbol{K} & \boldsymbol{F} \\
\boldsymbol{H} & \boldsymbol{H} & \boldsymbol{I} & \boldsymbol{F} & \boldsymbol{K} \\
\boldsymbol{K} & \boldsymbol{H} & \boldsymbol{F} & \boldsymbol{I} & \boldsymbol{H} \\
\boldsymbol{F} & \boldsymbol{F} & \boldsymbol{K} & \boldsymbol{H} & \boldsymbol{I}
\end{array}
$$

We will call this group the $A C C$ (Antithesis - Correspondent - Cofactor) group. The ACC group has the same structure of the Antithesis group. From its Cayley table, we see (i) that the correspondent of a price index coincides with the cofactor of its factor antithesis, (ii) that the cofactor of a price index coincides with the correspondent of its factor antithesis and (iii) that the factor antithesis of a price index coincides with the cofactor of its correspondent (or the correspondent of its cofactor, since the operators $\boldsymbol{H}$ and $\boldsymbol{K}$ commute). We will repeatedly employ these relationships in the following, discussing the properties of geo-logarithmic indexes.

\subsection{Crossings of a price index}

There is a standard technique to build a formula that fulfills the reversibility axioms (V) and (VI), starting from a formula that does not (Fisher, 1922; 
Martini, 2003; Fattore \& Quatto, 2004). Let us define two crossing operators $\otimes^{B}$ and $\otimes_{F}$, whose action on a price index formula is given by:

$$
\begin{aligned}
& \otimes^{B} P\left(\boldsymbol{p}_{a}, \boldsymbol{p}_{b}, \boldsymbol{q}_{a}, \boldsymbol{q}_{b}\right)=\sqrt{P\left(\boldsymbol{p}_{a}, \boldsymbol{p}_{b}, \boldsymbol{q}_{a}, \boldsymbol{q}_{b}\right) \cdot \boldsymbol{B} P\left(\boldsymbol{p}_{a}, \boldsymbol{p}_{b}, \boldsymbol{q}_{a}, \boldsymbol{q}_{b}\right)} \\
& \otimes_{F} P\left(\boldsymbol{p}_{a}, \boldsymbol{p}_{b}, \boldsymbol{q}_{a}, \boldsymbol{q}_{b}\right)=\sqrt{P\left(\boldsymbol{p}_{a}, \boldsymbol{p}_{b}, \boldsymbol{q}_{a}, \boldsymbol{q}_{b}\right) \cdot \boldsymbol{F} P\left(\boldsymbol{p}_{a}, \boldsymbol{p}_{b}, \boldsymbol{q}_{a}, \boldsymbol{q}_{b}\right)}
\end{aligned}
$$

By construction, $\otimes^{B} P$ and $\otimes_{F} P$ are invariant under the actions of $\boldsymbol{B}$ and $\boldsymbol{F}$ respectively. Since the crossing operators $\otimes^{B}$ and $\otimes_{\boldsymbol{F}}$ commute, the operator $\otimes_{F}^{B}=\otimes^{B} \circ \otimes_{F}=\otimes^{F} \circ \bigotimes_{B}$ can be defined as

$$
\bigotimes_{F}^{B} P=\sqrt{P \cdot \boldsymbol{B} P \cdot \boldsymbol{F} P \cdot \boldsymbol{D} P} .
$$

$\otimes_{F}^{B} P$ is invariant under the simultaneous action of $\boldsymbol{B}$ and $\boldsymbol{F}$, so it satisfies the basis reversibility and the factor reversibility axioms. The three crossing operators together with the identity operator form a monoid (Bosch, 2003) with the following Cayley table:

$$
\begin{array}{c|cc} 
& \boldsymbol{I} & \otimes^{B} \otimes_{F} \otimes_{F}^{B} \\
\hline \boldsymbol{I} & \boldsymbol{I} & \otimes^{B} \otimes_{F} \otimes_{F}^{B} \\
\otimes^{B} & \otimes^{B} \otimes^{B} \otimes_{F}^{B} \otimes_{F}^{B} \\
\otimes_{F} & \otimes_{F} \otimes_{F}^{B} \otimes_{F} \otimes_{F}^{B} \\
\otimes_{F}^{B} & \otimes_{F}^{B} \otimes_{F}^{B} \otimes_{F}^{B} \otimes_{F}^{B}
\end{array}
$$

\section{The geo-logarithmic family}

\subsection{The geo-logarithmic decomposition}

The starting point for the definition of the geo-logarithmic family is the geologarithmic decomposition of the value index $V$ (Martini, 2003), that we state without proof.

Proposition 1 Let $\boldsymbol{p}_{a}, \boldsymbol{p}_{b}, \boldsymbol{q}_{a}$ and $\boldsymbol{q}_{b}$ be the price and quantity vectors of situations $a$ and $b$ and let

$$
V=\frac{\sum_{i=1}^{n} p_{a i} q_{a i}}{\sum_{i=1}^{n} p_{b i} q_{b i}}
$$

be the value index. Then the following geo-logarithmic decomposition holds 
true:

$$
V=\prod_{i=1}^{n}\left(\frac{p_{a i}}{p_{b i}}\right)^{\frac{\tau\left(w_{a i}, w_{b i}\right)}{\sum_{j=1}^{n} \tau\left(w_{a j}, w_{b j}\right)}} \cdot \prod_{i=1}^{n}\left(\frac{q_{a i}}{q_{b i}}\right)^{\frac{\tau\left(w_{a i}, w_{b i}\right)}{\sum_{j=1}^{n} \tau\left(w_{a j}, w_{b j}\right)}}
$$

where

$$
w_{a i}=\frac{p_{a i} q_{a i}}{\sum_{i=1}^{n} p_{a i} q_{a i}}, \quad w_{b i}=\frac{p_{b i} q_{b i}}{\sum_{i=1}^{n} p_{b i} q_{b i}} \quad i=1, \ldots, n
$$

and

$\tau\left(w_{a i}, w_{b i}\right)$ is the logarithmic mean $\Lambda\left(w_{a i}, w_{b i}\right)$ of $w_{a i}$ and $w_{b i}$, defined for $w_{a i}, w_{b i}>0$ as:

$$
\Lambda\left(w_{a i}, w_{b i}\right)=\frac{w_{a i}-w_{b i}}{\ln w_{a i}-\ln w_{b i}}
$$

if $w_{a i} \neq w_{b i}$ and $\Lambda\left(w_{a i}, w_{b i}\right)=w_{a i}$, if $w_{a i}=w_{b i}$ (Carlson, 1972).

Note that

$$
\prod_{i=1}^{n}\left(\frac{p_{a i}}{p_{b i}}\right)^{\frac{\tau\left(w_{a i}, w_{b i}\right)}{\sum_{j=1}^{n} \tau\left(w_{a j}, w_{b j}\right)}}
$$

and

$$
\prod_{i=1}^{n}\left(\frac{q_{a i}}{q_{b i}}\right) \frac{\tau\left(w_{a i}, w_{b i}\right)}{\sum_{j=1}^{n} \tau\left(w_{a j}, w_{b j}\right)}
$$

are, respectively, the Sato-Vartia price index and the Sato-Vartia quantity index (Sato, 1976; Vartia, 1976).

\subsection{Definition of the geo-logarithmic family}

For $x, y \in[0,1]$, let $\boldsymbol{q}_{x}$ and $\boldsymbol{q}_{y}$ be two vectors, whose components are defined by

$$
q_{x i}=q_{a i}^{x} q_{b i}^{1-x}, \quad q_{y i}=q_{a i}^{y} q_{b i}^{1-y} \quad i=1, \ldots, n
$$

and let

$$
w_{x i}=\frac{p_{a i} q_{x i}}{\sum_{i=1}^{n} p_{a i} q_{x i}}, \quad w_{y i}=\frac{p_{b i} q_{y i}}{\sum_{i=1}^{n} p_{b i} q_{y i}} \quad i=1, \ldots, n
$$

The geo-logarithmic, or the $\mathrm{P}_{x y}$, family is the class of price indexes defined by:

$$
P_{x y}\left(\boldsymbol{p}_{a}, \boldsymbol{p}_{b}, \boldsymbol{q}_{a}, \boldsymbol{q}_{b}\right)=\prod_{i=1}^{n}\left(\frac{p_{a i}}{p_{b i}}\right)^{\frac{\tau\left(w_{x i}, w_{y i}\right)}{\sum_{j=1}^{n} \tau\left(w_{x j}, w_{y j}\right)}} .
$$


In the following, to ease the notation we will write

$$
P_{x y}\left(\boldsymbol{p}_{a}, \boldsymbol{p}_{b}, \boldsymbol{q}_{a}, \boldsymbol{q}_{b}\right)=\prod_{i=1}^{n}\left(\frac{p_{a i}}{p_{b i}}\right)^{\tau_{x y i} / \sum_{j=1}^{n} \tau_{x y j}}
$$

where $\tau_{x y i}=\tau\left(w_{x i}, w_{y i}\right)$.

The following proposition states a fundamental result about the $\mathrm{P}_{x y}$ parametrization:

Proposition 2 The mapping associating the pair $(x, y) \in[0,1] \times[0,1]$ to the index $P_{x y}$ is one to one, thus if $(x, y) \neq(u, v)$, then $P_{x y} \neq P_{u v}$.

Proof. See Appendix C.

The geo-logarithmic family can be introduced in a slightly different way. Let us indicate with $P_{10}\left(\boldsymbol{p}_{a}, \boldsymbol{p}_{b}, \boldsymbol{q}_{a}, \boldsymbol{q}_{b}\right)$ the Sato-Vartia price index. Then, we can define a $P_{x y}$ index by

$$
P_{x y}\left(\boldsymbol{p}_{a}, \boldsymbol{p}_{b}, \boldsymbol{q}_{a}, \boldsymbol{q}_{b}\right)=P_{10}\left(\boldsymbol{p}_{a}, \boldsymbol{p}_{b}, \boldsymbol{q}_{x}, \boldsymbol{q}_{y}\right) \quad x, y \in[0,1]
$$

where $\boldsymbol{q}_{x}$ and $\boldsymbol{q}_{y}$ are defined by means of (24). In other words, the members of the $\mathrm{P}_{x y}$ family are just the Sato-Vartia index computed on the virtual quantity vectors $\boldsymbol{q}_{x}$ and $\boldsymbol{q}_{y}$.

For future reference, note that the maps

$$
\begin{aligned}
\boldsymbol{T}_{x y} & : \mathbb{R}^{+n} \times \mathbb{R}^{+n} \mapsto \mathbb{R}^{+n} \times \mathbb{R}^{+n} \\
& :\left(\boldsymbol{q}_{a}, \boldsymbol{q}_{b}\right) \rightarrow\left(\boldsymbol{q}_{x}, \boldsymbol{q}_{y}\right)
\end{aligned}
$$

defined for $x, y \in[0,1]$ are invertible if and only if $x \neq y$, the inverse maps being (Fattore, 2006a)

$$
\boldsymbol{T}_{x y}^{-1}\left(\boldsymbol{q}_{x}, \boldsymbol{q}_{y}\right)=\left(\hat{\boldsymbol{q}}_{a}, \hat{\boldsymbol{q}}_{b}\right)
$$

where

$$
\hat{q}_{a i}=q_{x i}^{\frac{y-1}{y-x}} q_{y i}^{\frac{x-1}{x-y}} \quad i=1, \ldots, n
$$

and

$$
\hat{q}_{b i}=q_{x i}^{\frac{y}{y-x}} q_{y i}^{\frac{x}{x-y}} \quad i=1, \ldots, n .
$$

\subsection{Examples of geo-logarithmic price indexes}

The geo-logarithmic family contains some well known price indexes. Trivially, $P_{10}$ is the Sato-Vartia price index. On the other hand, if $x=y$ a few com- 
putations show that the formula of a geo-logarithmic price index simplifies in (Martini, 1992a)

$$
P_{x x}=\frac{\sum_{i=1}^{n} p_{a i} q_{x i}}{\sum_{i=1}^{n} p_{b i} q_{x i}}
$$

(for an explicit proof, see Appendix A). Thus the $\mathrm{P}_{x x}$ subfamily is composed of expenditure ratios and it is easily verified that $P_{00}$ is the Laspeyres price index, $P_{11}$ is the Paasche price index and $P_{0.50 .5}$ is the Walsh price index.

\section{Axiomatic properties of the geo-logarithmic family}

In this section the axiomatic properties fulfilled by geo-logarithmic price indexes are discussed. It is first proved that the elements of the $\mathrm{P}_{x y}$ family fulfill the proportionality, commensurability and homogeneity axioms and that they are basis reversible if and only if $y=1-x$ (Martini, 1992a). Then, it is shown that the monotonic geo-logarithmic price indexes are characterized by the condition $x=y$ and that the Sato-Vartia index is the only element of the $\mathrm{P}_{x y}$ family being factor reversible.

Proposition 3 Geo-logarithmic price indexes satisfy (i) the proportionality axiom, (ii) the commensurability axiom (iii) the homogeneity axiom. Moreover, they are basis reversible if and only if $y=1-x$.

Proof. (i) Let $\alpha$ be a strictly positive real number, then:

$$
P_{x y}\left(\boldsymbol{p}_{a}, \boldsymbol{p}_{b}, \boldsymbol{q}_{a}, \boldsymbol{q}_{b}\right)=\prod_{i=1}^{n} \alpha^{\tau_{x y i} / \sum_{j=1}^{n} \tau_{x y j}}=\alpha \quad x, y \in[0,1]
$$

thus the proportionality axiom is fulfilled by each element of the $\mathrm{P}_{x y}$ family; (ii) let $U$ be a $n \times n$ diagonal matrix of strictly positive weights. For every $x, y \in[0,1]$ and every $i=1, \ldots, n$, the ratios $p_{a i} / p_{b i}$ and the coefficients $w_{x i}$ and $w_{y i}$ are invariant under the simultaneous transformations $\boldsymbol{p}_{a} \rightarrow U \boldsymbol{p}_{a}$, $\boldsymbol{p}_{b} \rightarrow U \boldsymbol{p}_{b}, \boldsymbol{q}_{a} \rightarrow U^{-1} \boldsymbol{q}_{a}$ and $\boldsymbol{q}_{b} \rightarrow U^{-1} \boldsymbol{q}_{b}$, thus any geo-logarithmic index fulfills the commensurability axiom; (iii) let $\alpha$ and $\beta$ be two strictly positive real numbers. For every $i=1, \ldots, n$ and every $x, y \in[0,1]$, both $w_{x i}$ and $w_{y i}$ are invariant under the transformations $\boldsymbol{p}_{a} \rightarrow \alpha \boldsymbol{p}_{a}$ and $\boldsymbol{p}_{b} \rightarrow \beta \boldsymbol{p}_{b}$, thus

$$
P_{x y}\left(\alpha \boldsymbol{p}_{a}, \beta \boldsymbol{p}_{b}, \boldsymbol{q}_{a}, \boldsymbol{q}_{b}\right)=\prod_{i=1}^{n}\left(\frac{\alpha p_{a i}}{\beta p_{b i}}\right)^{\frac{\tau\left(w_{x i}, w_{y i}\right)}{\sum_{j=1}^{n} \tau\left(w_{x j}, w_{y j}\right)}}=\frac{\alpha}{\beta} P_{x y}\left(\boldsymbol{p}_{a}, \boldsymbol{p}_{b}, \boldsymbol{q}_{a}, \boldsymbol{q}_{b}\right)
$$

and the homogeneity axiom is fulfilled by geo-logarithmic price indexes; (iv) requiring $\boldsymbol{B} P_{x y}=P_{x y}$ is the same as requiring $P_{1-y 1-x}=P_{x y}$ or $y=1-x$, by virtue of Proposition 2. We will indicate the class of basis reversible geologarithmic price index by $\mathrm{P}_{x 1-x}$. 
When introduced, geo-logarithmic price indexes were supposed to be monotonic (Martini, 1992a). Later, the Sato-Vartia price index was proved not to be monotonic (Reinsdorf \& Dorfman, 1999). Using this result, the following proposition shows that a geo-logarithmic index is monotonic if and only if it belongs to the subfamily $\mathrm{P}_{x x}$ of the expenditure ratios.

Proposition 4 An element of the $\mathrm{P}_{x y}$ family is monotonic if and only if $x=$ $y$.

Proof. Let

$$
P_{x y}\left(\boldsymbol{p}_{a}, \boldsymbol{p}_{b}, \boldsymbol{q}_{a}, \boldsymbol{q}_{b}\right)=P_{10}\left(\boldsymbol{p}_{a}, \boldsymbol{p}_{b}, \boldsymbol{q}_{x}, \boldsymbol{q}_{y}\right) \quad x, y \in[0,1]
$$

be an element of the geo-logarithmic family. As shown in Section 3.2, if $x \neq y$, the map $\boldsymbol{T}_{x y}$ is invertible, thus $P_{x y}$ is not monotonic since $P_{10}$ is not. If $x=y$, (35) simplifies in

$$
P_{x x}=\frac{\sum_{i=1}^{n} p_{a i} q_{x i}}{\sum_{i=1}^{n} p_{b i} q_{x i}}
$$

which is clearly monotonic for every $x \in[0,1]$.

Let us now address the problem of factor reversibility of geo-logarithmic price indexes. The Sato-Vartia index is factor reversible, but it is trivial to verify that in general geo-logarithmic price indexes are not (consider, for example, the Laspeyres, Paasche and Walsh indexes). The following proposition shows that the class of factor reversible geo-logarithmic price indexes does reduce to the Sato-Vartia index only:

Proposition 5 The only element of the $\mathrm{P}_{x y}$ family satisfying the factor reversibility axiom is the Sato-Vartia index.

Proof. The proof is by contradiction. Let $x, y \in[0,1]$ and put $Q_{x y}=\operatorname{Cor} P_{x y}$ to ease the notation. If $P_{x y}$ satisfies the factor reversibility axiom, then for any choice of the price and quantity vectors, the following identity holds:

$$
P_{x y}\left(\boldsymbol{p}_{a}, \boldsymbol{p}_{b}, \boldsymbol{q}_{a}, \boldsymbol{q}_{b}\right) \cdot Q_{x y}\left(\boldsymbol{p}_{a}, \boldsymbol{p}_{b}, \boldsymbol{q}_{a}, \boldsymbol{q}_{b}\right)=\frac{\sum_{i=1}^{n} p_{a i} q_{a i}}{\sum_{i=1}^{n} p_{b i} q_{b i}} .
$$

Since the Sato-Vartia index $P_{10}$ does satisfy the factor reversibility axiom, (37) can be equivalently written as:

$$
P_{x y}\left(\boldsymbol{p}_{a}, \boldsymbol{p}_{b}, \boldsymbol{q}_{a}, \boldsymbol{q}_{b}\right) \cdot Q_{x y}\left(\boldsymbol{p}_{a}, \boldsymbol{p}_{b}, \boldsymbol{q}_{a}, \boldsymbol{q}_{b}\right)=P_{10}\left(\boldsymbol{p}_{a}, \boldsymbol{p}_{b}, \boldsymbol{q}_{a}, \boldsymbol{q}_{b}\right) \cdot Q_{10}\left(\boldsymbol{p}_{a}, \boldsymbol{p}_{b}, \boldsymbol{q}_{a}, \boldsymbol{q}_{b}\right)
$$


for any choice of the price and quantity vectors. Let us consider the price vectors $\boldsymbol{p}_{a}^{*}=(p, 1, \ldots, 1)$, where $p \neq 1$ and $p>0$, and $\boldsymbol{p}_{b}^{*}=(1,1, \ldots, 1)$ and let $\boldsymbol{q}_{a}^{*}=\boldsymbol{p}_{a}^{*}$ and $\boldsymbol{q}_{b}^{*}=\boldsymbol{p}_{b}^{*}$. Corollary 12 in Appendix C shows that for every fixed $x, y \in[0,1],(x, y) \neq(1,0), p$ can be chosen in such a way that

$$
P_{x y}\left(\boldsymbol{p}_{a}^{*}, \boldsymbol{p}_{b}^{*}, \boldsymbol{q}_{a}^{*}, \boldsymbol{q}_{b}^{*}\right) \neq P_{10}\left(\boldsymbol{p}_{a}^{*}, \boldsymbol{p}_{b}^{*}, \boldsymbol{q}_{a}^{*}, \boldsymbol{q}_{b}^{*}\right) .
$$

On the other hand, it is immediate to check that for every $x, y \in[0,1]$

$$
P_{x y}\left(\boldsymbol{p}_{a}^{*}, \boldsymbol{p}_{b}^{*}, \boldsymbol{q}_{a}^{*}, \boldsymbol{q}_{b}^{*}\right)=Q_{x y}\left(\boldsymbol{p}_{a}^{*}, \boldsymbol{p}_{b}^{*}, \boldsymbol{q}_{a}^{*}, \boldsymbol{q}_{b}^{*}\right)
$$

so that (37) gives:

$$
P_{x y}\left(\boldsymbol{p}_{a}^{*}, \boldsymbol{p}_{b}^{*}, \boldsymbol{q}_{a}^{*}, \boldsymbol{q}_{b}^{*}\right)^{2}=P_{10}\left(\boldsymbol{p}_{a}^{*}, \boldsymbol{p}_{b}^{*}, \boldsymbol{q}_{a}^{*}, \boldsymbol{q}_{b}^{*}\right)^{2}
$$

or

$$
P_{x y}\left(\boldsymbol{p}_{a}^{*}, \boldsymbol{p}_{b}^{*}, \boldsymbol{q}_{a}^{*}, \boldsymbol{q}_{b}^{*}\right)=P_{10}\left(\boldsymbol{p}_{a}^{*}, \boldsymbol{p}_{b}^{*}, \boldsymbol{q}_{a}^{*}, \boldsymbol{q}_{b}^{*}\right)
$$

since price indexes are certainly positive.

Clearly, (42) and (39) are incompatible.

\section{Global properties of the geo-logarithmic family and its subfami- lies}

In the previous section, we have discussed the axiomatic properties of geologarithmic price indexes. Now, we investigate some global properties of the $\mathrm{P}_{x y}$ family and its main subfamilies $\mathrm{P}_{x x}$ and $\mathrm{P}_{x 1-x}$. Particularly, we discuss the closure of these classes under the action of the Antithesis group and the Crossing monoid. For technical reasons, it is convenient to start studying the properties of the $\mathrm{P}_{x x}$ and the $\mathrm{P}_{x 1-x}$ subfamilies.

\subsection{The $\mathrm{P}_{x x}$ subfamily}

As already shown, price indexes belonging to the $\mathrm{P}_{x x}$ subfamily fulfill axioms (I) - (IV). Since

$$
\mathrm{P}_{x x} \cap \mathrm{P}_{x 1-x}=\left\{P_{0.50 .5}\right\}
$$

the Walsh price index is the only monotonic geo-logarithmic index being basis reversible. Nevertheless, the $\mathrm{P}_{x x}$ subfamily is closed under the action of the basis antithesis operator. In fact, from $\boldsymbol{B} P_{x y}=P_{1-y 1-x}$, it follows $\boldsymbol{B} P_{x x}=$ 
$P_{1-x 1-x} \in \mathrm{P}_{x x}$. On the contrary, neither a factor reversible element of $\mathrm{P}_{x x}$ exists, nor this class is closed under the action of the factor antithesis operator. The first assertion is trivial, since the only factor reversible geo-logarithmic index is $P_{10}$; the second assertion is proved in the following proposition:

Proposition 6 Let $P_{x x} \in \mathrm{P}_{x x}$. $\boldsymbol{F} P_{x x}$ satisfies the axiom of monotonicity if and only if $x=0$ or $x=1$. In other words, the only monotonic elements of the $\mathrm{P}_{x y}$ family having monotonic factor antithesis are the Laspeyres and the Paasche indexes.

Proof. A direct computation shows that

$$
\boldsymbol{F} P_{x x}=\frac{\sum_{i=1}^{n} p_{a i} q_{a i}}{\sum_{i=1}^{n} p_{b i} q_{b i}} \cdot \frac{\sum_{i=1}^{n} q_{b i}\left(p_{b i}\right)^{x}\left(p_{a i}\right)^{1-x}}{\sum_{i=1}^{n} q_{a i}\left(p_{b i}\right)^{x}\left(p_{a i}\right)^{1-x}} .
$$

After some algebraic computations, the derivative of (44) with respect to $p_{a j}$ is obtained as

$$
\frac{\partial}{\partial p_{a j}} \boldsymbol{F} P_{x x}=\frac{U+V \cdot Z}{T}
$$

where

$$
\begin{aligned}
T & =\sum_{i=1}^{n} p_{b i} q_{b i} \sum_{i=1}^{n} q_{a i} p_{b i}^{x} p_{a i}^{1-x} \\
U & =q_{a j} \sum_{i=1}^{n} q_{b i} p_{b i}^{x} p_{a i}^{1-x} \\
V & =(1-x) p_{b j}^{x} p_{a j}^{-x} \sum_{i=1}^{n} p_{a i} q_{a i} \\
Z & =q_{b j}-\frac{\sum_{i=1}^{n} q_{b i} p_{b i}^{x} p_{a i}^{1-x}}{\sum_{i=1}^{n} q_{a i} p_{b i}^{x} p_{a i}^{1-x}} q_{a j} .
\end{aligned}
$$

If $x=0$ or $x=1$, the derivative is certainly positive, for any choice of price and quantity vectors, since $\boldsymbol{F} P_{00}$ is the Paasche index and $\boldsymbol{F} P_{11}$ is the Laspeyres index (both strictly monotonic price indexes). If $0<x<1$, the derivative is negative when

$$
Z<-\frac{U}{V}
$$

and is positive when $Z>-\frac{U}{V}$. Explicitly, condition (50) becomes

$$
\frac{q_{b j}}{q_{a j}}<\frac{\sum_{i=1}^{n} q_{b i} p_{b i}^{x} p_{a i}^{1-x}}{\sum_{i=1}^{n} q_{a i} p_{b i}^{x} p_{a i}^{1-x}}-\frac{\sum_{i=1}^{n} q_{b i} p_{b i}^{x} p_{a i}^{1-x}}{(1-x) p_{b j}^{x} p_{a j}^{-x} \sum_{i=1}^{n} p_{a i} q_{a i}} .
$$

For any fixed $x \in(0,1)$, this inequality can be indeed satisfied by a suitable choice of $\boldsymbol{p}_{a}, \boldsymbol{p}_{b}, \boldsymbol{q}_{a}$ and $\boldsymbol{q}_{b}$ (for an explicit proof, see Appendix D). 
From Proposition (6), we can easily derive some further results about the $\mathrm{P}_{x x}$ subfamily, collected in the following corollary:

Corollary 7 (i) The $\mathrm{P}_{x x}$ subfamily is not closed under the action of operator $\boldsymbol{D}$; (ii) the only fixed points of $\boldsymbol{D}$ within the subfamily $\mathrm{P}_{x x}$ are the Laspeyres and the Paasche indexes; (iii) the $\mathrm{P}_{x x}$ subfamily is not closed under the action of operator $\boldsymbol{F}$, the only $\mathrm{P}_{x x}$ indexes whose factor antithesis is in $\mathrm{P}_{x x}$ being the Laspeyres and the Paasche indexes.

Proof. (i) Since $\boldsymbol{B} \mathrm{P}_{x x}=\mathrm{P}_{x x}$, then, from Proposition (6), $\boldsymbol{D} \mathrm{P}_{x x}=\boldsymbol{F} \mathrm{P}_{x x} \nsubseteq \mathrm{P}_{x x}$, i.e. $\mathrm{P}_{x x}$ is not closed under the action of $\boldsymbol{D}$; (ii) If $\boldsymbol{D} P_{x x}=P_{x x}$, then $\boldsymbol{B} P_{x x}=$ $\boldsymbol{F} P_{x x} \in \mathrm{P}_{x x}$; since $\boldsymbol{B} P_{x x} \in \mathrm{P}_{x x}$, then, from Proposition (4), $\boldsymbol{F} P_{x x}$ is monotonic and necessarily $x=0$ or $x=1$; (iii) for $x \neq 0,1, \boldsymbol{F} P_{x x}$ is not monotonic, then it cannot belong to the $\mathrm{P}_{x x}$ subfamily, by virtue of Proposition (6). On the other hand, $\boldsymbol{F} P_{00}=P_{11}$ and $\boldsymbol{F} P_{11}=P_{00}$.

Finally, it is trivial to show that the $\mathrm{P}_{x x}$ subfamily is not closed under the action of the crossing operators. In fact, the Fisher index, which is not a geologarithmic index (it is factor reversible), can be obtained applying $\otimes^{B}, \otimes_{F}$, or $\otimes_{F}^{B}$ to $P_{00}$ (Laspeyres price index) or to $P_{11}$ (Paasche price index).

\subsection{The $\mathrm{P}_{x 1-x}$ subfamily}

The elements of the $\mathrm{P}_{x 1-x}$ subfamily satisfy axioms (I)-(III) and axiom (V). In general, they violate axiom (IV), the only monotonic element of this subfamily being the Walsh price index, and axiom (VI), the only factor reversible element being the Sato-Vartia price index. Moreover, the $\mathrm{P}_{x 1-x}$ subfamily is not closed under the action of the factor antithesis operator, as the following proposition proves.

Proposition 8 The only element of the $\mathrm{P}_{x 1-x}$ subfamily whose factor antithesis belongs to the subfamily $\mathrm{P}_{x 1-x}$ is the Sato-Vartia index.

Proof. If $\boldsymbol{F} P_{x 1-x} \in \mathrm{P}_{x 1-x}$, then $\boldsymbol{F} P_{x 1-x}=P_{u 1-u}$ for some $u \in[0,1]$. Hence, in particular, the Taylor series of $\boldsymbol{F} P_{x 1-x}$ and $P_{u 1-u}$ must coincide, in the neighborhood of any point. Choose the price and quantity vectors as follows 


$$
\begin{aligned}
& \boldsymbol{p}_{a}^{*}=(1+\varepsilon, 1, \ldots, 1) \\
& \boldsymbol{p}_{b}^{*}=(1,1, \ldots, 1) \\
& \boldsymbol{q}_{a}^{*}=(1+\varepsilon, 1, \ldots, 1) \\
& \boldsymbol{q}_{b}^{*}=(1,1, \ldots, 1) .
\end{aligned}
$$

Appendix B shows that the condition for the Taylor series to coincide is given by

$$
6-3 n-n^{2}+\left(2-3 n+n^{2}\right) x^{2}=10-9 n+n^{2}+\left(-2+3 n-n^{2}\right) u^{2}
$$

or

$$
x=\sqrt{2-u^{2}} \quad x, u \in[0,1]
$$

whose only acceptable solution is $x=u=1$.

From the above discussion, it follows immediately that the $\mathrm{P}_{x 1-x}$ subfamily is not closed under the action of the crossing operators $\otimes_{F}$ and $\otimes_{F}^{B}$, while it is trivially closed under the action of the crossing $\otimes_{B}$, since its elements are basis reversible.

Besides the Sato-Vartia index, there is another interesting element of the $\mathrm{P}_{x 1-x}$ subfamily, namely $P_{01}$, which after Martini can be named Crossing Values (Martini, 1992a). This index is remarkable not only for the axiomatic properties it fulfills, but also because it provides an alternative way to obtain the Fisher index. In fact, it can be directly shown that the Fisher index equals the crossing between $P_{01}$ and its factor antithesis:

$$
P^{f s h}=\otimes_{F}\left(P_{01}\right)=\sqrt{P_{01} \cdot \boldsymbol{F}\left(P_{01}\right)} .
$$

\subsection{Closure properties of the geo-logarithmic family}

The geo-logarithmic family is not closed under the action of the Antithesis group. In fact, while $\boldsymbol{B} P_{x y} \in \mathrm{P}_{x y}$, the same is not true with regard to the operator $\boldsymbol{F}$ (and hence with regard to the operator $\boldsymbol{D}$ too).

Proposition 9 The geo-logarithmic family is not closed under the action of the factor antithesis operator.

Proof. The proof is by contradiction. Let us suppose that $\mathrm{P}_{x y}$ is closed under the action of $\boldsymbol{F}$. Let $x \neq 0$ and consider an element of the subfamily $\mathrm{P}_{x 1-x}$. Since $\boldsymbol{B}$ and $\boldsymbol{F}$ commute, we have

$$
\boldsymbol{B} \boldsymbol{F} P_{x 1-x}=\boldsymbol{F} \boldsymbol{B} P_{x 1-x}=\boldsymbol{F} P_{x 1-x}
$$


which implies $\boldsymbol{F} P_{x 1-x} \in \mathrm{P}_{x 1-x}$. Thus, the subfamily $\mathrm{P}_{x 1-x}$ is closed under the action of $\boldsymbol{F}$, but this is false, as shown by Proposition 8 .

We conclude this paragraph, noting that the geo-logarithmic family is not closed under the action of the Crossing monoid, since the Fisher index is not a geo-logarithmic index, as previously shown.

\section{Properties of the cofactors of geo-logarithmic price indexes}

As already pointed out, the choice of a specific price index induces the choice of its cofactor as the associated quantity index, in the decomposition (2) of the value index; for this reason, it is of interest to investigate the axiomatic properties satisfied by the cofactors of geo-logarithmic price indexes. From the general theory of price index discussed at the beginning of this paper, we know that the cofactor of a price index fulfills the commensurability axiom, the basis reversibility axiom and the factor reversibility axiom, if and only if the price index itself does. It then follows immediately that the cofactor of a geo-logarithmic price index $P_{x y}$ satisfies (i) the commensurability axiom for every $x, y \in[0,1]$, (ii) the basis reversibility axiom if and only if $y=1-x$ and (iii) the factor reversibility axiom if and only if $x=1$ and $y=0$ (i.e. if and only if $P_{x y}$ is the Sato-Vartia price index).

The following proposition shows that cofactors of geo-logarithmic price indexes satisfy also the proportionality and homogeneity axioms (Martini, 1992a, 2003).

Proposition 10 Let $P_{x y} \in \mathrm{P}_{x y}$, then Cof $P_{x y}$ satisfies (i) the proportionality axiom and (ii) the homogeneity axiom.

Proof. (i) Let $\alpha$ be a strictly positive real number and let $\boldsymbol{q}_{a}=\alpha \boldsymbol{q}_{b}$. The vectors of virtual quantities $\boldsymbol{q}_{x}$ and $\boldsymbol{q}_{y}$, defined in (24), are simply

$$
\boldsymbol{q}_{x}=\alpha^{x} \boldsymbol{q}_{b}, \quad \boldsymbol{q}_{y}=\alpha^{y} \boldsymbol{q}_{b}
$$

and it is immediate that in this case $w_{x i}$ and $w_{y i}$ coincide with $w_{a i}$ and $w_{b i}$, as defined in (20). Hence, we can write

$$
P_{x y}\left(\boldsymbol{p}_{a}, \boldsymbol{p}_{b}, \alpha \boldsymbol{q}_{b}, \boldsymbol{q}_{b}\right)=\prod_{i=1}^{n}\left(\frac{p_{a i}}{p_{b i}}\right)^{\frac{\tau\left(w_{a i}, w_{b i}\right)}{\sum_{j=1}^{n} \tau\left(w_{a i}, w_{b i}\right)}} \quad x, y \in[0,1] .
$$

Substituting (61) in (3) and using (19), we obtain 


$$
\begin{aligned}
& \operatorname{Cof} P_{x y}\left(\boldsymbol{p}_{a}, \boldsymbol{p}_{b}, \alpha \boldsymbol{q}_{b}, \boldsymbol{q}_{b}\right)= \\
& =\frac{\prod_{i=1}^{n}\left(\frac{p_{a i}}{p_{b i}}\right)^{\frac{\tau\left(w_{a i}, w_{b i}\right)}{\sum_{j=1}^{n} \tau\left(w_{a i}, w_{b i}\right)}} \cdot \prod_{i=1}^{n}\left(\frac{\alpha q_{b i}}{q_{b i}}\right)^{\frac{\tau\left(w_{a i}, w_{b i}\right)}{\sum_{j=1}^{n} \tau\left(w_{a i}, w_{b i}\right)}}}{\prod_{i=1}^{n}\left(\frac{p_{a i}}{p_{b i}}\right)^{\frac{\tau\left(w_{a i}, w_{b i}\right)}{\sum_{j=1}^{n}\left(w_{a i}, w_{b i}\right)}}}=\alpha .
\end{aligned}
$$

(ii) Let $\alpha$ and $\beta$ be two strictly positive real numbers. Since the functions $w_{x i}$ and $w_{y i}$, defined in (25), are left unchanged by the transformations $\boldsymbol{q}_{a} \rightarrow \alpha \boldsymbol{q}_{a}$ and $\boldsymbol{q}_{b} \rightarrow \beta \boldsymbol{q}_{b}$, it follows that $P_{x y}\left(\boldsymbol{p}_{a}, \boldsymbol{p}_{b}, \alpha \boldsymbol{q}_{a}, \beta \boldsymbol{q}_{b}\right)=P_{x y}\left(\boldsymbol{p}_{a}, \boldsymbol{p}_{b}, \boldsymbol{q}_{a}, \boldsymbol{q}_{b}\right)$. Thus

$$
\begin{aligned}
& \operatorname{Cof} P_{x y}\left(\boldsymbol{p}_{a}, \boldsymbol{p}_{b}, \alpha \boldsymbol{q}_{a}, \beta \boldsymbol{q}_{b}\right)= \\
= & \frac{\alpha}{\beta} \cdot \frac{\sum_{i=1}^{n} p_{a i} q_{a i}}{P_{x y}\left(\boldsymbol{p}_{a}, \boldsymbol{p}_{b}, \boldsymbol{q}_{a}, \boldsymbol{q}_{b}\right) \cdot \sum_{i=1}^{n} p_{b i} q_{b i}}=\frac{\alpha}{\beta} \cdot \operatorname{Cof} P_{x y}\left(\boldsymbol{p}_{a}, \boldsymbol{p}_{b}, \boldsymbol{q}_{a}, \boldsymbol{q}_{b}\right) .
\end{aligned}
$$

Finally, we discuss the axiom of monotonicity. In general, the cofactors of geologarithmic indexes violate the monotonicity axiom. Consider, for example, the cofactor of the Sato-Vartia price index. Due to factor reversibility, its cofactor coincides with its correspondent which is not monotonic with respect to quantities, since the Sato-Vartia price index itself is non monotonic with respect to prices. The following proposition shows that monotonic cofactors are an exception even if we restrict ourselves to monotonic geo-logarithmic price indexes:

Proposition 11 The only elements of the $\mathrm{P}_{x x}$ subfamily whose cofactors satisfy the monotonicity axiom are the Laspeyres and the Paasche indexes.

Proof. The correspondent of a price index is monotonic with respect to quantities if and only if the price index itself is monotonic with respect to prices. The thesis now follows from Proposition 6, since the cofactor of a price index is just the correspondent of its factor antithesis.

We conclude this Section with a comment. It is very easily shown that the antithesis and the crossing operators preserve proportionality, commensurability and homogeneity of both geo-logarithmic price indexes and their cofactors. We have previously proved that the geo-logarithmic family is not closed under the action of the Antithesis group and the Crossing monoid. This means that applying these sets of operators to $\mathrm{P}_{x y}$ a wider family $\overline{\mathrm{P}}_{x y}$ is obtained whose elements do share proportionality, commensurability and homogeneity with their cofactors. This new family is obviously closed under the action of the 
Antithesis group and the Crossing monoid and, as will be briefly discussed in the Conclusion, it seems to play a very important role when the joint axiomatic consistency of a price index and its cofactor is concerned.

\section{Geo-logarithmic and superlative price indexes}

Following an economic approach to the price index problem, Diewert proposed two parametric families of price indexes, that he called superlative in his conceptual framework (Diewert, 1976). Although the axiomatic and the economic approaches are very different in nature, it is worth studying the connection between geo-logarithmic and superlative price indexes. It turns out that the only superlative index number belonging to the geo-logarithmic family is the Walsh index. The proof of this fact is rather long and can be found in Appendix $\mathrm{E}$, where we prove a little more. In fact, we show that there are only three superlative price indexes being proportional together with their cofactors, namely the Walsh index, its factor antithesis and the Fisher index. Since proportionality of the price index and its cofactor is a necessary condition for the price index itself to belong to the geo-logarithmic family, the conclusion follows immediately from the results we have proved in the previous paragraphs.

Among superlative price indexes, a very important role is played by the Törnqvist formula (Balk \& Diewert, 2001):

$$
P^{t r n}=\prod_{i=1}^{n}\left(\frac{p_{a i}}{p_{b i}}\right)^{\left(w_{a i}+w_{b i}\right) / 2} .
$$

The Törnqvist index does not belong to the $\mathrm{P}_{x y}$ family, since its cofactor is not proportional. Neither it can be obtained from geo-logarithmic price indexes, by means of the Antithesis group or the Crossing monoid. This can be proved in different ways, but the easiest one is considering that such sets of operators preserve proportionality of geo-logarithmic price indexes and their cofactors. Nevertheless, an interesting relation can be stated between the Törnqvist index and the geo-logarithmic family. In Appendix $\mathrm{F}$ we show that in the limit $w_{\text {axi }} \rightarrow w_{\text {byi }}(i=1, \ldots, n)$, it holds

$$
P_{x y} \approx \prod_{i=1}^{n}\left(\frac{p_{a i}}{p_{b i}}\right)^{\left(w_{a x i}+w_{b y i}\right) / 2} .
$$

Thus, the first order approximation of a geo-logarithmic price index has a Törnqvist-like form. In the case of the Sato-Vartia price index, i.e. for $x=1$ and $y=0,(65)$ gives the Törnqvist formula. 


\section{Conclusion}

In this paper we have presented the geo-logarithmic family of price indexes, discussing its axiomatic properties. It has been shown that all geo-logarithmic indexes are proportional, commensurable and homogeneous, together with their cofactors. Geo-logarithmic indexes satisfying the axioms of monotonicity, basis reversibility and factor reversibility have been identified and their properties have been investigated. Properties of the geo-logarithmic family and of its main subfamilies under the action of the Antithesis group and the Crossing monoid have been studied. The relationship between the geo-logarithmic family and Diewert's superlative price indexes has also been investigated. However, some issues remain open; for example, the consistency-in-aggregation problem is still to be faced. Recently, an interesting approach to consistencyin-aggregation has appeared (Pursiainen, 2005) that seems to be very effective and some of our further research will be devoted to apply it to geo-logarithmic price indexes.

As discussed in the introduction and along the paper, the geo-logarithmic family seems to play a central role when joint consistent comparisons of both prices and quantities are concerned. On one hand, all geo-logarithmic price indexes share the properties of proportionality, commensurability and homogeneity with their cofactors; on the other hand, all known price indexes sharing such properties with their cofactors belong to the geo-logarithmic family or to its image $\overline{\mathrm{P}}_{x y}$ under the action of the Antithesis group or the Crossing monoid. (A direct check on known price index formulas shows that usually it is the axiom of proportionality that is not fulfilled by cofactors of non geo-logarithmic price indexes; in a few cases, it is the axiom of homogeneity that is violated). We are tempted to conjecture that this is true in full generality and to affirm that a cofactor of a proportional, commensurable and homogeneous price index fulfills these same three properties with respect to quantities, if and only if the price index itself belongs to $\bar{P}_{x y}$. So far, we could not prove the validity of such a general statement. Nevertheless, investigating this issue more deeply could be very interesting; more generally, it could be valuable both at theoretical and applied level to investigate throughly the relationships between the axiomatic properties of a price index and the axiomatic properties of its cofactor and to find out conditions assuring the joint consistency of price and quantity comparisons.

\section{A Proof of identity (32)}

Proof. If $x=y$, then obviously according to $(24) q_{x i}=q_{y i}, \forall i=1, \ldots, n$. Substituting for $q_{a i}=q_{b i}=q_{x i}$ in (18) and (19) and taking into account (27), 
we obtain

$$
\frac{\sum_{i=1}^{n} p_{a i} q_{x i}}{\sum_{i=1}^{n} p_{b i} q_{x i}}=\prod_{i=1}^{n}\left(\frac{p_{a i}}{p_{b i}}\right)^{\frac{\tau_{x x i}}{\sum_{i=1}^{n} \tau_{x x i}}} \cdot \prod_{i=1}^{n}\left(\frac{q_{x i}}{q_{x i}}\right)^{\frac{\tau_{x x i}}{\sum_{i=1}^{n} \tau_{x x i}}}
$$

Observing that the last factor is simply 1, we get

$$
\frac{\sum_{i=1}^{n} p_{a i} q_{x i}}{\sum_{i=1}^{n} p_{b i} q_{x i}}=\prod_{i=1}^{n}\left(\frac{p_{a i}}{p_{b i}}\right)^{\frac{\tau_{x x i}}{\sum_{i=1}^{n} \tau_{x x i}}}=P_{x x}\left(\boldsymbol{p}_{a}, \boldsymbol{p}_{b}, \boldsymbol{q}_{a}, \boldsymbol{q}_{b}\right)
$$

\section{B Taylor expansions}

This appendix reports the Taylor series of the logarithm of geo-logarithmic price indexes needed in the previous discussion about the axiomatic properties of geo-logarithmic family. The computations involved in the Taylor expansions are very lengthly and were performed with the aid of a computer software. All the Taylor series considered have been computed for the following choice of price and quantity vectors:

$$
\begin{aligned}
& \boldsymbol{p}_{a}^{*}=(1+\varepsilon, 1, \ldots, 1) \\
& \boldsymbol{p}_{b}^{*}=(1,1, \ldots, 1) \\
& \boldsymbol{q}_{a}^{*}=(1+\varepsilon, 1, \ldots, 1) \\
& \boldsymbol{q}_{b}^{*}=(1,1, \ldots, 1)
\end{aligned}
$$

$\varepsilon$ being arbitrarily small. This particular choice easies the computations and is just what is needed to prove the results discussed in the paper.

\section{B.1 Taylor series for $\ln P_{x y}$}

Let $x, y \in[0,1]$. Substituting the vectors $\boldsymbol{p}_{a}^{*}, \boldsymbol{p}_{b}^{*}, \boldsymbol{q}_{a}^{*}, \boldsymbol{q}_{a}^{*}$ into (26), we obtain the following expression for the logarithm of a geo-logarithmic index:

$$
\ln P_{x y}\left(\boldsymbol{p}_{a}^{*}, \boldsymbol{p}_{b}^{*}, \boldsymbol{q}_{a}^{*}, \boldsymbol{q}_{b}^{*}\right)=\frac{\tau_{x y 1}^{*}}{\tau_{x y 1}^{*}+(n-1) \tau_{x y}^{*}} \ln (1+\varepsilon)
$$

where

$$
\tau_{x y 1}^{*}=\Lambda\left(\frac{(1+\varepsilon)^{1+x}}{(1+\varepsilon)^{1+x}+n-1}, \frac{(1+\varepsilon)^{y}}{(1+\varepsilon)^{y}+n-1}\right)
$$


and

$$
\tau_{x y}^{*}=\Lambda\left(\frac{1}{(1+\varepsilon)^{1+x}+n-1}, \frac{1}{(1+\varepsilon)^{y}+n-1}\right) .
$$

The Taylor series of $\ln P_{x y}$, up to order 3 in $\varepsilon$ turns out to be

$$
\ln P_{x y} \approx \frac{1}{n} \varepsilon+A(x, y) \varepsilon^{2}+B(x, y) \varepsilon^{3}
$$

where

$$
\begin{aligned}
A(x, y)= & \frac{-1+(n-1)(x+y)}{2 n^{2}} \\
B(x, y)= & \frac{2+\left(2-3 n+n^{2}\right)\left(x^{2}+x y+y^{2}\right)}{6 n^{3}}+ \\
& -\frac{2\left(n^{2}-1\right) y+\left(4-3 n-n^{2}\right) x}{6 n^{3}} .
\end{aligned}
$$

\section{B.2 Taylor series for $\ln P_{x 1-x}$ and $\ln \boldsymbol{F} P_{x 1-x}$}

With the choice (A.1) - (A.4) of price and quantity vectors, the Taylor expansions for the logarithms of a geo-logarithmic index belonging to the subfamily $\mathrm{P}_{x 1-x}$ and its factor antithesis are obtained as

$$
\ln P_{x y} \approx \frac{1}{n} \varepsilon+\frac{n-2}{2 n^{2}} \varepsilon^{2}+\frac{6-3 n-n^{2}+\left(2-3 n+n^{2}\right) x^{2}}{6 n^{3}} \varepsilon^{3}
$$

and

$$
\ln \boldsymbol{F} P_{x y} \approx \frac{1}{n} \varepsilon+\frac{n-2}{2 n^{2}} \varepsilon^{2}+\frac{10-9 n+n^{2}+\left(-2+3 n-n^{2}\right) x^{2}}{6 n^{3}} \varepsilon^{3} .
$$

\section{Proof of Proposition 2}

We will prove Proposition 2, showing that if $(x, y) \neq(u, v)$, then the Taylor series (B.8) of $\ln P_{x y}$ and $\ln P_{u v}$ are different. Let $n \geq 2$ and let $u, v \in[0,1]$ be fixed. Choose $x \in[0,1]$ and $y \in[0,1]$ such that $P_{x y}=P_{u v}$. If the price and quantity vectors are chosen as in (A.1) - (A.4), then from (B.8) the equality $P_{x y}=P_{u v}$ implies the conditions (i) $A(x, y)=A(u, v)$ and (ii) $B(x, y)=$ 
$B(u, v)$. From (B.9), condition (i) gives

$$
\frac{-1+(n-1)(x+y)}{2 n^{2}}=\frac{-1+(n-1)(u+v)}{2 n^{2}}
$$

or

$$
y=u+v-x .
$$

Taking into account (B.10) and (C.1), after some algebraic computations condition (ii) becomes

$$
\frac{\left(2-3 n+n^{2}\right)(x-u)(1-v+x)}{6 n^{3}}=0
$$

i.e. $x=u$ or $x=v-1$. If $x=u$, then from (C.1) it is $y=v$. If $x=v-1$, then $v=1$, (since $x, v \in[0,1]$ ), but then necessarily $x=0$ and, again from (C.1), $y=u+1$. The latter equality implies $u=0$ (otherwise $y \notin[0,1]$ ) and $y=1$. Hence, in either cases, $x=u$ and $y=v$.

The following corollary, needed in the proof of Proposition 5, is now easily proved.

Corollary 12 Let $\boldsymbol{p}_{a}^{*}=(p, 1, \ldots, 1)$ and $\boldsymbol{p}_{b}^{*}=(1,1, \ldots, 1)(p \neq 1, p>0)$. Let $\boldsymbol{q}_{a}^{*}=\boldsymbol{p}_{a}^{*}$ and $\boldsymbol{q}_{b}^{*}=\boldsymbol{p}_{b}^{*}$. Then $p$ exists such that

$$
P_{x y}\left(\boldsymbol{p}_{a}^{*}, \boldsymbol{p}_{b}^{*}, \boldsymbol{q}_{a}^{*}, \boldsymbol{q}_{b}^{*}\right) \neq P_{10}\left(\boldsymbol{p}_{a}^{*}, \boldsymbol{p}_{b}^{*}, \boldsymbol{q}_{a}^{*}, \boldsymbol{q}_{b}^{*}\right)
$$

for every $x, y \in[0,1]$ such that $(x, y) \neq(1,0)$.

Proof. Proof of Proposition 2 shows that if $x \neq u$ or $y \neq v$ then $P_{x y}\left(\boldsymbol{p}_{a}^{*}, \boldsymbol{p}_{b}^{*}, \boldsymbol{q}_{a}^{*}, \boldsymbol{q}_{b}^{*}\right)$ and $P_{u v}\left(\boldsymbol{p}_{a}^{*}, \boldsymbol{p}_{b}^{*}, \boldsymbol{q}_{a}^{*}, \boldsymbol{q}_{b}^{*}\right)$, as functions of $p$, differ in a neighborhood of $p=1$. In particular, this holds if $u=1$ and $v=0$. Thus $p$ exists such that condition (C.3) is satisfied.

\section{Proof of inequality (51)}

In this appendix, we prove that inequality (51) in the proof of Proposition 6 can indeed be satisfied, for any fixed $x \in(0,1)$, by a suitable choice of price and quantity vectors. 
Let

$$
W\left(\boldsymbol{p}_{a}, \boldsymbol{p}_{b}, \boldsymbol{q}_{a}, \boldsymbol{q}_{b}\right)=\frac{1}{\sum_{i=1}^{n} q_{a i} p_{b i}^{x} p_{a i}^{1-x}}-\frac{1}{(1-x) p_{b j}^{x} p_{a j}^{-x} \sum_{i=1}^{n} p_{a i} q_{a i}},
$$

and write (51) as

$$
\frac{q_{b j}}{q_{a j}}<W\left(\boldsymbol{p}_{a}, \boldsymbol{p}_{b}, \boldsymbol{q}_{a}, \boldsymbol{q}_{b}\right) \cdot \sum_{i=1}^{n} q_{b i} p_{b i}^{x} p_{a i}^{1-x} .
$$

Let $p_{a j}=p_{b j}=\lambda / q_{a j}$ and $p_{b i}=(1-x)^{k / x} p_{a i}(i \neq j)$, where $\lambda$ and $k$ are strictly positive constants and let the other price and quantity components be fixed. For such a choice of the prices, (D.2) simplifies in

$$
\frac{q_{b j}}{q_{a j}}<\left[\lambda \frac{q_{b j}}{q_{a j}}+\sum_{i \neq j} q_{b i} p_{a i}(1-x)^{k}\right] \cdot W^{*}\left(\boldsymbol{p}_{a}, \boldsymbol{q}_{a} ; \lambda, k\right)
$$

where

$$
W^{*}\left(\boldsymbol{p}_{a}, \boldsymbol{q}_{a} ; \lambda, k\right)=\frac{1}{\lambda+(1-x)^{k} \sum_{i \neq j}^{n} q_{a i} p_{a i}}-\frac{1}{(1-x) \lambda+(1-x) \sum_{i \neq j}^{n} p_{a i} q_{a i}}
$$

does not depend upon $q_{a j}$ and $q_{b j}$.

Since

$$
\lim _{k \rightarrow \infty} W^{*}\left(\boldsymbol{p}_{a}, \boldsymbol{q}_{a} ; \lambda, k\right)=\frac{1}{\lambda}-\frac{1}{(1-x) \lambda+(1-x) \sum_{i \neq j}^{n} p_{a i} q_{a i}},
$$

if $\lambda_{x}$ is chosen so that

$$
\lambda_{x}<\frac{1-x}{x} \sum_{i \neq j}^{n} p_{a i} q_{a i}
$$

then $\lim _{k \rightarrow \infty} W^{*}\left(\boldsymbol{p}_{a}, \boldsymbol{q}_{a} ; \lambda_{x}, k\right)>0$. Hence, if $k_{x}$ is chosen large enough, it is certainly $W^{*}\left(\boldsymbol{p}_{a}, \boldsymbol{q}_{a} ; \lambda_{x}, k_{x}\right)>0$. As a consequence, we have

$$
L_{x}=\lim _{q_{b j} / q_{a j} \rightarrow 0}\left(\lambda_{x} \frac{q_{b j}}{q_{a j}}+\sum_{i \neq j} q_{b i} p_{a i}(1-x)^{k_{x}}\right) \cdot W^{*}\left(\boldsymbol{p}_{a}, \boldsymbol{q}_{a} ; \lambda_{x}, k_{x}\right)>0 .
$$

Hence if $q_{b j} / q_{a j}$ is small enough, inequality (D.3) (and thus (51)) is satisfied.

\section{E Proportional superlative index numbers with proportional co- factors}

Diewert proposed two families $P^{(r)}$ and $\hat{P}^{(r)}$ of superlative index numbers, whose members are defined as follows (Hill, 2006) 


$$
P^{(r)}=\left[\frac{\sum_{i=1}^{n} p_{b i}^{\frac{2-r}{r}} p_{a i}^{\frac{r}{2}} q_{b i}}{\sum_{i=1}^{n} p_{b i} q_{b i}} / \frac{\sum_{i=1}^{n} p_{a i}^{\frac{2-r}{r}} p_{b i}^{\frac{r}{2}} q_{a i}}{\sum_{i=1}^{n} p_{a i} q_{a i}}\right]^{1 / r}
$$

and

$$
\hat{P}^{(r)}=\left[\frac{\sum_{i=1}^{n} q_{a i}^{\frac{2-r}{r}} q_{b i}^{\frac{r}{2}} p_{a i}}{\sum_{i=1}^{n} q_{b i}^{\frac{2-r}{r}} q_{a i}^{\frac{r}{2}} p_{b i}}\right]^{1 / r} .
$$

with $r \neq 0$. It is easily checked that $P_{a b}^{(r)}$ and $\hat{P}_{a b}^{(r)}$ are the factor antithesis of each other. The family $P_{a b}^{(r)}$ can be extended taking the limit $r \rightarrow 0$, which gives the Törnqvist price index

$$
P^{t r n}=P^{(0)}=\prod_{i=1}^{n}\left(\frac{p_{a i}}{p_{b i}}\right)^{\left(w_{a i}+w_{b i}\right) / 2}
$$

where $w_{a i}$ and $w_{b i}$ are defined as in (20).

In this section, we show that the only superlative index numbers having proportional cofactors are the Walsh index, its factor antithesis and the Fisher index.

Rearranging terms in (E.1), the general expression for $P_{a b}^{(r)}$ can be cast in the form

$$
P_{a b}^{(r)}=V^{\frac{1}{r}}\left[\frac{\sum_{i=1}^{n} p_{a i}^{\frac{2-r}{r}} p_{b i}^{\frac{r}{2}} q_{a i}}{\sum_{i=1}^{n} p_{b i}^{\frac{2-r}{r}} p_{a i}^{\frac{r}{2}} q_{b i}}\right]^{-\frac{1}{r}}
$$

so that the cofactor of $P_{a b}^{(r)}$ is easily given by

$$
\operatorname{Cof}\left(P^{(r)}\right)=\frac{V}{P^{(r)}}=V^{\frac{r-1}{r}}\left[\frac{\sum_{i=1}^{n} p_{a i}^{\frac{2-r}{r}} p_{b i}^{\frac{r}{2}} q_{a i}}{\sum_{i=1}^{n} \cdot p_{b i}^{\frac{2-r}{r}} p_{a i}^{\frac{r}{2}} q_{b i}}\right]^{\frac{1}{r}}
$$

If $\operatorname{Cof}\left(P^{(r)}\right)$ is to be proportional, then for $\boldsymbol{q}_{a}=\boldsymbol{q}_{\boldsymbol{b}}=(1, \ldots, 1)$

$$
\left(\frac{\sum_{i=1}^{n} p_{a 1}}{\sum_{i=1}^{n} p_{b 1}}\right)^{r-1}\left[\frac{\sum_{i=1}^{n} p_{a i}^{\frac{2-r}{r}} p_{b i}^{\frac{r}{2}}}{\sum_{i=1}^{n} p_{b i}^{\frac{2-r}{r}} p_{a i}^{\frac{r}{2}}}\right]=1
$$

holds for any choice of the price vectors. Particularly, if we set $p_{b i}=1 \forall i=$ $1, \ldots, n$, the above expression furtherly simplifies in

$$
\frac{1}{n^{r-1}}\left(\sum_{i=1}^{n} p_{a i}\right)^{r-1} \cdot \frac{\sum_{i=1}^{n} p_{a i}^{\frac{2-r}{2}}}{\sum_{i=1}^{n} p_{a i}^{\frac{r}{2}}}=1
$$


or

$$
\left(\sum_{i=1}^{n} p_{a i}\right)^{r-1} \cdot \sum_{i=1}^{n} p_{a i}^{\frac{2-r}{2}}=n^{r-1} \sum_{i=1}^{n} p_{a i}^{\frac{r}{2}}
$$

and this must hold for any choice of the price vector $\boldsymbol{p}_{a}$.

Putting $\boldsymbol{p}_{a}=(1+\varepsilon, 1, \ldots, 1)$ in the above identity we obtain

$$
(n+\varepsilon)^{r-1}\left(n-1+(1+\varepsilon)^{\frac{2-r}{2}}\right)=n^{r-1}\left(n-1+(1+\varepsilon)^{\frac{r}{2}}\right)
$$

and expanding for $\varepsilon$ arbitrarily small gives

$$
n^{r-1}\left(1+(r-1) \frac{\varepsilon}{n}\right)\left(n+\frac{2-r}{2} \varepsilon\right)=n^{r-1}\left(n+\frac{r}{2} \varepsilon\right) .
$$

Simplifying and rearranging terms, we get

$$
\frac{(r-1)(2-r) \varepsilon^{2}}{2 n}=0 .
$$

Thus $r=1$ or $r=2$ are necessary conditions for $\operatorname{Cof}\left(P^{(r)}\right)$ to satisfy the proportionality axiom. For $r=1$, the superlative index $P^{(1)}$ is easily seen to be the factor antithesis of the Walsh price index, or $\boldsymbol{F}\left(P_{0.50 .5}\right)$ in terms of the geo-logarithmic parametrization. Since the cofactor of the Walsh price index satisfies the proportionality axiom, it follows easily that also the factor antithesis of the Walsh index does. For $r=2$, the superlative index $P_{a b}^{(1)}$ is nothing but the Fisher index, whose cofactor clearly satisfies the proportionality axiom. We can conclude that $P_{a b}^{(r)}$ has proportional cofactor if and only if $r=1$ or $r=2$. In neither cases, such indexes belong to the geo-logarithmic family.

Let us now consider the family $\hat{P}^{(r)}$. Since $\hat{P}^{(r)}$ is just the factor antithesis of $P^{(r)}$, it follows that proportionality of $\hat{P}^{(r)}$ (a necessary condition to belong to the geo-logarithmic family) is the same as proportionality of $\boldsymbol{F}\left(P^{(r)}\right)$. In turn, proportionality (with respect to prices) of $\boldsymbol{F}\left(P^{(r)}\right)$ is equivalent to proportionality (with respect to quantities) of $\operatorname{Cor}\left(\boldsymbol{F}\left(P^{(r)}\right)\right)=\operatorname{Cof}\left(P^{(r)}\right)$. From the above discussion it follows that $\hat{P}^{(r)}$ is proportional if and only if $r=1$ or $r=2$, giving the Walsh price index $(r=1)$ and (again) the Fisher price index $(r=2)$. In both cases, the cofactors are proportional. Finally, it is immediate to check that the Törnqvist index, which is proportional, has a cofactor which is not. 


\section{F Proof of approximation (65)}

For $\eta>0$ arbitrarily small, let

$$
w_{a x i}=w_{b y i}+\varepsilon_{i} \quad i=1, \ldots, n
$$

with $\left|\varepsilon_{i}\right| \leq \eta(i=1, \ldots, n)$ under the constraint

$$
\sum_{i=1}^{n} \varepsilon_{i}=0
$$

so that $\sum_{i=1}^{n} w_{a x i}=1$.

Let us first consider the Taylor expansion of $\tau\left(w_{b y i}+\varepsilon_{i}, w_{b y i}\right)$ :

$$
\begin{aligned}
\tau\left(w_{\text {byi }}+\varepsilon_{i}, w_{\text {byi }}\right) & =\frac{\varepsilon_{i}}{\ln \left(1+\varepsilon_{i} / w_{\text {byi }}\right)}= \\
& =\frac{\varepsilon_{i}}{\varepsilon_{i} / w_{\text {byi }}-1 / 2 \cdot\left(\varepsilon_{i} / w_{\text {byi }}\right)^{2}+o\left(\varepsilon_{i}^{2}\right)}= \\
& =\frac{w_{\text {byi }}}{1-1 / 2 \cdot \varepsilon_{i} / w_{\text {byi }}+o\left(\varepsilon_{i}\right)}= \\
& =w_{\text {byi }}\left(1+\frac{1}{2} \frac{\varepsilon_{i}}{w_{b y i}}+o\left(\varepsilon_{i}\right)\right)= \\
& =w_{\text {byi }}+\frac{1}{2} \varepsilon_{i}+o\left(\varepsilon_{i}\right) .
\end{aligned}
$$

Using this result and (F.2), we have

$$
\sum_{i=1}^{n} \tau\left(w_{\text {byi }}+\varepsilon_{i}, w_{\text {byi }}\right)=\sum_{i=1}^{n}\left(w_{\text {byi }}+\frac{1}{2} \varepsilon_{i}+o\left(\varepsilon_{i}\right)\right)=1+\sum_{i=1}^{n} o\left(\varepsilon_{i}\right)
$$

so that

$$
\frac{\tau_{i}\left(w_{b y i}+\varepsilon_{i}, w_{b y i}\right)}{\sum_{i=1}^{n} \tau_{i}\left(w_{b y i}+\varepsilon_{i}, w_{b y i}\right)}=\frac{w_{b y i}+\frac{1}{2} \varepsilon_{i}+o\left(\varepsilon_{i}\right)}{1+\sum_{i=1}^{n} o\left(\varepsilon_{i}\right)}
$$

Inserting this expansion into (26) and taking the first order approximation, after some computation we obtain

$$
\prod_{i=1}^{n}\left(\frac{p_{a i}}{p_{b i}}\right)^{\tau_{i} / \sum_{i=1}^{n} \tau_{i}} \approx \prod_{i=1}^{n}\left(\frac{p_{a i}}{p_{b i}}\right)^{w_{b y i}+\frac{1}{2} \varepsilon_{i}}
$$

where as usual $\tau_{i}=\tau_{i}\left(w_{a x i}, w_{b y i}\right)$. Using (F.1) in the above expression, we get (65). 


\section{References}

Balk B. M. (1995). Axiomatic Price Index Theory: A Survey, International Statistical Review, 63, 69-93.

Balk B. M. \& Diewert W. E. (2001). A characterization of the Törnqvist price index, Economics Letters, Volume 72, Issue 3, 279-281

Bosch S. (2003). Algebra, Springer-Verlag, Heidelberg.

Carlson B. C. (1972). The logarithmic mean, Amer. Math. Monthly, 79, 615618.

Diewert W. E. (1976). Exact and superlative index numbers, Journal of Econometrics 4, 115-145.

Diewert W. E. (1992). Essays in Index Number Theory: an Overview of Volume 1, Discussion Paper No.: 92-31, The University of British Columbia, Vancouver.

Eichhorn W. \& Voeller J. (1990). Axiomatic foundations of price indexes and purchasing power parities, Price level measurement, North-holland, Amsterdam.

Eichhorn W. \& Voeller J. (1976a). Theory of the Price Indices, Lecture Notes in Economics and Mathematical Systems, Springer-Verlag, Berlin.

Eichhorn W. \& Voeller J. (1976b). Theory of the price index: Fisher's test approach and generalisations. Lectures notes in economics and mathematical systems, Springer-Verlag, Berlin.

Fattore M. (2006a). On the monotonicity of the geo-logarithmic price indexes, Società Italiana di Statistica, Proceedings of the XLIII Scientific Meeting, Cleup, Padova.

Fattore M. (2006b). Finite Group in Axiomatic Index Number Theory, Department of Statistics, University of Milan - Bicocca (available at http://econpapers.repec.org).

Fattore M. \& Quatto P. (2004). Strutture algebriche dei numeri indice bilaterali, in Studi in onore di Marco Martini, Giuffré, Milan.

Fisher I. (1922). The making of index numbers, Houghton Mifflin, Boston.

Hill R. J. (2006). Superlative index numbers: not all of them are super, Journal of Econometrics, 130, 25-43.

Krstcha M. (1988). Axiomatic characterization of statistical price indices, Physica-Verlag, Heidelberg.

IMF (2004). Producer Price Index Manual, International Monetary Fund.

Martini M. (2003). Numeri indice per il confronto nel tempo e nello spazio, Cusl, Milan.

Martini M. (1992a). I numeri indice in un approccio assiomatico, Giuffré, Milan.

Martini M. (1992b). General Function of Axiomatic Index Numbers. J. Ital. Statist. Soc., 3359 - 376.

Miller W. (1972). Symmetry Groups and Their Applications, Academic Press, New York.

Pursiainen H. (2005). Consistent Aggregation Methods and Index Number 
Theory, Academic Dissertation, Faculty of Social Sciences, University of Helsinki.

Reinsdorf M. B. \& Dorfman A. H. (1999). The Sato-Vartia index and the monotonicity axiom, Journal of Econometrics, 90, 45-61.

Sato K. (1976). The ideal log-change index number, The review of Economics and Statistics, 58, n. 2, 223-228.

Swamy S. (1965). Consistency of Fisher's tests, Econometrica, 33, 619-623.

Tung W. (1972). Group theory in physics, World Scientific Publishing Co., Singapore.

Vogt A. \& Barta J. (1997). The Making of Tests for Index Numbers, PhysicaVerlag, Heidelberg.

Vartia Y. O. (1976). Ideal log-change index numbers, Scandinavian Journal of Statistics, 3, 121-126. 